\title{
Coulisses
}

Revue de théâtre

6 | Printemps 1992

Varia

\section{La Famille Schroffenstein}

\section{Emmanuelle Ravot}

\section{OpenEdition}

\section{Journals}

Édition électronique

URL : http://journals.openedition.org/coulisses/1894

DOI : $10.4000 /$ coulisses. 1894

ISSN : 2546-9460

\section{Éditeur}

Presses universitaires de Franche-Comté

\section{Édition imprimée}

Date de publication : 1 juin 1992

ISSN : 1150-594X

\section{Référence électronique}

Emmanuelle Ravot, « La Famille Schroffenstein », Coulisses [En ligne], 6 | Printemps 1992, mis en ligne le 15 mars 2019, consulté le 10 décembre 2020. URL : http://journals.openedition.org/coulisses/1894 ; DOI : https://doi.org/10.4000/coulisses.1894

Ce document a été généré automatiquement le 10 décembre 2020.

Coulisses 


\title{
La Famille Schroffenstein
}

\author{
Emmanuelle Ravot
}

La famille Schroffenstein, pièce de Kleist traduite et mise en scène par Eloi Recoing, a été créée à la Métaphore de Lille, puis représentée à Montpellier et au C.D.N. de Besançon (11 au 19 mars 92).

\section{Le metteur en scène :}

1 Si ce n'est un père marionnettiste, rien ne prédestinait Eloi Recoing au théâtre. Après son baccalauréat, il se dirige vers des études de médecine qu'il abandonne au bout de quatre ans pour s'orienter vers la philosophie. C'est au contact des penseurs et philosophes allemands qu'il apprend à apprécier la langue germanique. Il obtient un poste dans l'enseignement, mais une fois encore, il change de cap et prend une voie qui le conduira au théâtre. Attiré par le théâtre allemand, Eloi Recoing se rend en Allemagne, $y$ vit durant deux années et rencontre Ruth Orthmann qui deviendra sa femme et avec qui il co-traduira la pièce de Kleist.

2 Après avoir travaillé sept ans en tant qu'assistant d'Antoine Vitez, il décide, à la mort de ce dernier, de se consacrer à la mise en scène. Très impressionné par Kleist, étonné de constater que les œuvres de l'auteur allemand sont généralement peu ou mal traduites (toujours en prose et dans le registre romantique), Eloi Recoing entreprend un travail sur Die Familie Schroffenstein, pièce inédite en français. Les conditions de la traduction sont intéressantes puisqu'il travaille en collaboration avec Ruth Orthmann, tous deux étant parfaitement bilingues mais de langue maternelle différente. La pièce de Kleist est écrite en vers blancs, vers classique du théatre allemand qui n'a pas son équivalent en français. Pour conserver le caractère particulier de l'écriture originale, les deux traducteurs ont choisi une transposition en vers libre afin de garder « les effets d'enjambement ménagés par le poète ». Ils ont également respecté la ponctuation de Kleist, notamment l'usage du tiret, « véritable notation musicale des suspens du souffle, soupirs de la pensée qui cherche à dissiper l'incompréhensible ».

3 Pour Eloi Recoing, traduire, c'est déjà mettre en scène. Aussi la pièce présentée au C.D.N. fut en parfaite continuité avec le texte français et avec son auteur. En effet, 
l'introduction de marionnettes dans le spectacle n'est pas gratuite. Outre le fait qu'Eloi Recoing fut élevé dans un théâtre de marionnettes, ce "jouet d'enfant» avait également fortement influencé Kleist lui-même. Dans la représentation, la présence de marionnettes permet la distanciation et rend compte de la part onirique du spectacle.

4 Autre élément à rattacher au monde de l'enfance, le rideau mobile. Eloi Recoing voulait qu'il soit comme les pages d'un livre d'enfant que l'on tourne, comme les images d'un rêve. Cela permet aussi de ne pas "faire entrer" les comédiens, mais de les faire apparaître ou disparaitre comme sous l'effet d'une "ardoise magique » Enfin, il est important de noter une présence invisible, celle de Kleist, à travers la musique (solos de clarinette rappelant que Kleist fut un grand clarinettiste) qui est le fil conducteur, la respiration du spectacle.

5 Le public a été réceptif à Kleist et à la mise en scène de sa pièce. Les applaudissements ont peut-être donné l'envie à Eloi Recoing de monter d'autres pièces de Kleist pour mieux nous le faire connaître, en tous cas, nous l'espérons.

\section{L'auteur :}

Bernd Wilhelm Heinrich von Kleist est né le 18 octobre 1777 à Francfort-sur-l'Oder, dans une famille de grande aristocratie sans fortune. Par tradition, il envisage le métier des armes et, en juin 1792 - quatre ans après la mort de son père - s'engage dans le régiment des gardes de Potsdam et part pour la campagne du Rhin. Cependant, peu fait pour la carrière militaire, il quitte l'armée en 1799 pour se consacrer aux études à l'université de sa ville natale. A la fin de l'année 1800, il projette d'écrire un drame qu'il intitule La famille Ghonorez (histoire qui se déroule en Espagne) et, installé en Suisse, près du lac de Thun, il achève cette première œuvre. Mais, ayant la possibilité de monter la pièce au théâtre de Graz, spécialisé dans les pièces chevaleresques du MoyenAge, Kleist modifie le lieu et les noms de son drame. La pièce, qui s'intitule désormais $L a$ famille Schroffenstein, est jouée pour la première fois dans ce théâtre le 14 janvier 1804.

Kleist n'a pas vingt-cinq ans lorsqu'il écrit sa première pièce, et il doit subir la double dictature littéraire de Goethe et de Schiller. Pourtant, il réussira à échapper à l'influence de l'un comme de l'autre. Il s'inspirera plutôt de Rousseau, auteur qu'il admire, et de sa notion de contrat social. Pendant ses dix années d'existence littéraire, Kleist va beaucoup voyager et écrire (poèmes, essais, articles, nouvelles, drames...). Ses pièces seront généralement mal accueillies. La petite Catherine de Heilbronn (1808) est la seule œuvre de Kleist à avoir connu le succès du vivant de l'auteur.

8 Toute sa vie, l'idée de suicide ne quitte pas l'auteur et le 21 novembre 1811 il met fin à ses jours avec une amie, Henriette Vogel, au bord du Wannsee à Berlin. Avant de mourir, il écrit ces quelques lignes : «Ce n'est pas un malheur d'avoir perdu le bonheur, le seul malheur, c'est de ne plus se souvenir de soi-même ».

La famille Schroffenstein 


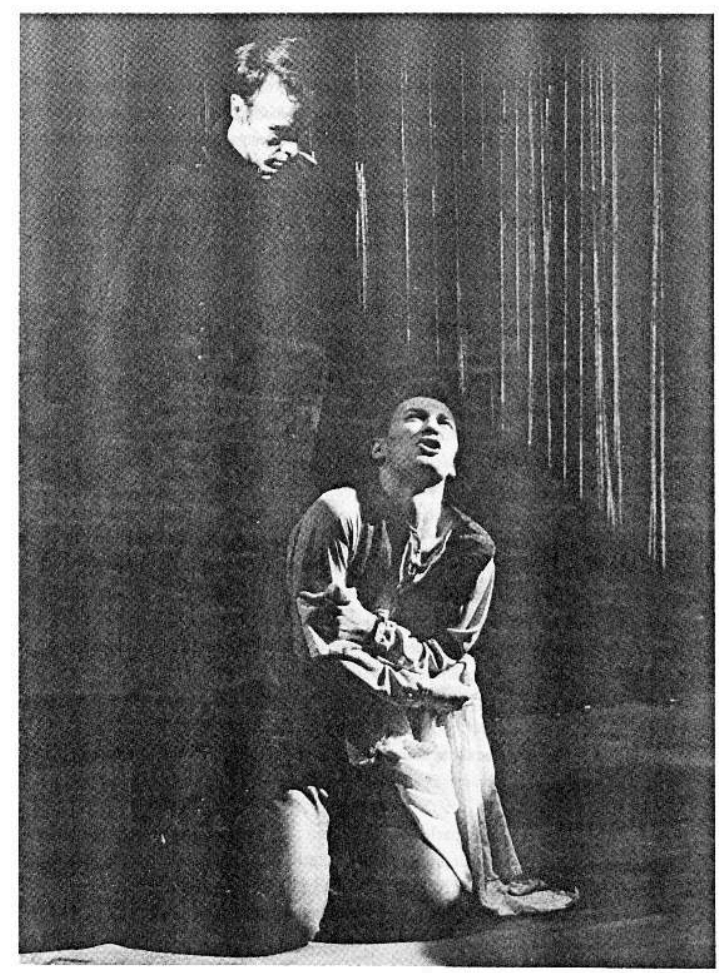

Photo Daniel Rapaich

\section{La pièce :}

La famille Schroffenstein pose le problème de la vengeance. Deux branches d'une même famille entrent dans une logique de guerre à la suite de la mort d'un enfant. Le soupçon, né d'un contrat d'héritage, va conduire cette famille à sa perte. Les termes du contrat sont énoncés au début de la pièce $(\mathrm{I}, 1)$ :

Depuis des temps anciens, existe entre nos deux maisons parentes

Rossitz et Warwand, un contrat d'héritage,

Lequel stipule que si l'une des branches

S'éteignait totalement, la totalité de son avoir

Reviendrait à l'autre.

11 Le drame commence avec le meurtre de Pierre, neuf ans, le plus jeune héritier de la branche Rossitz. Sont accusés deux hommes de la branche ennemie. Suit une lutte sans merci, ponctuée de morts, qui s'achève par le meurtre des enfants par les pères. Les deux chefs de clan, abusés par les apparences, tuent chacun leur propre enfant, anéantissant par leur haine aveugle toute descendance.

12 Selon Jean-Paul Gaillard et Jean-Paul Dromard, psychanalystes et spécialistes de Kleist, l'auteur de La famille Schroffenstein, dont l'inconscient est toujours sur le devant de la scène, est une mine d'or pour un psychanalyste. L'auteur ne décrit que lui-même et il n'y a aucun de ses héros qui ne soit lui-même. Pour J.-P. Gaillard, Kleist est celui qui annonce le plus clairement la psychanalyse et aujourd'hui encore, il a beaucoup à nous apprendre.

13 Le point de départ de la pièce est un contrat écrit par un ancêtre commun aux deux branches de la famille et, dès le début, Kleist nous oblige à croire au pouvoir maléfique 
de ce texte. Mais il y a également l'importance de la rumeur, qui transmet et accrédite le soupçon. Et c'est lui le véritable ennemi: les gens ne se parlent plus, ne se comprennent plus et le drame intervient.

14 Une question est posée par les psychanalystes : «Puisqu'il existe un lien de parenté entre les deux jeunes gens tués par leur propre père, le meurtre n'est-il pas ici l'envers de l'inceste?». En fait, les deux branches de la famille sont si proches qu'elles se brisent. Et, pour un psychanalyste, « le meurtre d'un enfant, c'est un inceste d'un autre temps ». 